\title{
Properties of matrices included in the calculation of geomagnetically induced currents (GICs) in power systems and introduction of a test model for GIC computation algorithms
}

\author{
Risto Pirjola \\ Finnish Meteorological Institute, P.O. Box 503, 00101 Helsinki, Finland \\ (Received April 1, 2008; Revised July 22, 2008; Accepted September 10, 2008; Online published February 18, 2009)
}

\begin{abstract}
"Geomagnetically induced currents" (GICs) in ground-based networks are a manifestation of space weather and a potential source of problems to the systems. Exact matrix equations, which are summarized in this paper, for calculating GICs in a power system were formulated in the 1980s. These equations are reformulated here to show how they lead to a direct relation between GICs and the horizontal geoelectric field. Properties of the matrices, which enable the derivation of physical features of the flow of the GICs in a power system, are considered in detail. A specific aim of this study was to show that the ratio of the GICs obtained at a station under two different geophysical situations is independent of the total earthing resistance at that particular station. The behavior of the elements of the transfer matrix between geovoltages and GICs confirms the earlier result that it is sufficient to make a calculation of a small grid around the site if the GIC values at one site are of interest. Using the developed technique, I have calculated the GICs of the old configuration of the Finnish 400-kV grid.
\end{abstract}

Key words: Geomagnetically induced current, GIC, power system, space weather, matrix calculation.

\section{Introduction}

"Geomagnetically induced currents" (GICs) flowing in networks, such as electric power transmission grids, oil and gas pipelines, telecommunication cables, and railway systems, are the ground end of "space weather" resulting from the activity of the Sun (e.g. Lanzerotti et al., 1999). The plasma physical and electromagnetic phenomena associated with space weather constitute a complicated chain of processes. Both space-borne and ground-based technology can experience problems due to space weather.

Despite the complexities of the processes in the space weather chain, the basic physical principle of the GICs is easily understandable based on Faraday's and Ohm's laws: rapidly changing currents in the Earth's magnetosphere and ionosphere during a space storm create temporal variations in the geomagnetic field that induce a (geo)electric field, which then drives currents in conducting materials. In addition to technological networks, the Earth itself is also a conductor. Thus, currents induced in the ground also contribute to the geomagnetic disturbance field and to the geoelectric field occurring at the Earth's surface (e.g. Watermann, 2007).

The effects of the GICs on technology were noted in telegraph systems as early as the 1840 s (e.g. Boteler et al., 1998; Lanzerotti et al., 1999; and references therein). In power systems, the direct current (dc)-like GIC may saturate transformers, possibly leading to problems that can even cause a collapse of the whole system or permanent

Copyright (c) The Society of Geomagnetism and Earth, Planetary and Space Sciences (SGEPSS); The Seismological Society of Japan; The Volcanological Society of Japan; The Geodetic Society of Japan; The Japanese Society for Planetary Sciences; TERRAPUB. damage to transformers (e.g. Kappenman and Albertson, 1990; Kappenman, 1996; Bolduc, 2002; Molinski, 2002; and references therein). Two well-known and documented events are the blackouts in Québec, Canada, in March 1989 (Bolduc, 2002) and southern Sweden in October 2003 (Pulkkinen et al., 2005).

The GICs caused by intense geomagnetic disturbances are a problem especially in high-latitude auroral regions, but the auroral oval usually moves towards much lower latitudes during major geomagnetic storms. The GIC amplitudes are also affected by the grid topology, configuration, and resistances. Furthermore, the sensitivities of systems to the GICs depend on many engineering details; for example, a small-amplitude GIC that does not at all disturb one grid may be problematic to another. These facts suggest that networks in lower latitudes may be affected by the GICs as well. The increasing sizes of high-voltage power grids, the complex interconnections, and the extensive transport of energy emphasize the importance of GIC issues at all latitudes (Kappenman, 2004).

A calculation of GICs in a given ground-based system is usually separated into two parts: (1) a "geophysical part", which encompasses the determination of the horizontal geoelectric field occurring at the Earth's surface; (2) an "engineering part", which covers the computation of GICs produced by the geoelectric field. The input of the geophysical part includes information on ground conductivity and data or assumptions about magnetospheric-ionospheric currents or about magnetic variations at the Earth's surface; the calculations are independent of the particular technological grid. The engineering part uses the geoelectric field and the network configuration and resistances as the input. 
Due to the low frequencies involved in geoelectromagnetic phenomena and GICs, a dc treatment is acceptable in the engineering part, in which the calculations can, in principle, be performed exactly, using the given connections and resistances of the system. In practice, however, an accurate description of all components affecting GICs in a complex network is difficult. This paper focuses on the engineering part of the GIC calculation in the case of a power system, which constitutes a discretely earthed network having earthings at transformer neutrals at (sub)stations.

Matrix equations that enable the calculation of GICs in a power grid were originally derived by Lehtinen and Pirjola (1985) and subsequently reported in many publications (e.g. Pirjola, 2005a, 2007, 2008a). These are summarized in Section 2 because they constitute the basis of the present study. In addition, a simple matrix equation is derived that couples GICs directly to the geoelectric field using the given basic formulas that include the (geo)voltages along the transmission lines associated with the geoelectric field.

In order to study the GICs in a system, the GICs at each site of the grid are usually first calculated under a uniform northward and a uniform eastward field of $1 \mathrm{~V} / \mathrm{km}$. The effects of the grid topology, configuration, and resistances on the GIC distribution are then revealed without the effects of complex electric field structures. In this way, sites that most likely may experience GIC problems are identified, and the locations of possible GIC recordings can be planned properly. The magnitude of $1 \mathrm{~V} / \mathrm{km}$ is a typical value during a geomagnetic storm, although larger values are also reported in the literature (see e.g. Pirjola and Lehtinen, 1985; and references therein). However, the values obtained for $1 \mathrm{~V} / \mathrm{km}$ are directly scalable to any other magnitude because GICs are linearly dependent on the geoelectric field. Similarly, GICs for a uniform horizontal geoelectric field of any direction are obtained as linear combinations from the results for an eastward and a northward field. The ratio of GICs for the northward and eastward cases is also a useful parameter because it gives the relative importance of the two geoelectric components for the GIC at a particular site affected by the grid configuration and resistances (see, for example, Wik et al., 2008). In Section 3, I show that the ratio of the earthing GICs at a station is independent of the earthing resistance at the particular station under two different geophysical situations, such as a uniform northward and eastward geoelectric field.

Although all computation techniques for the engineering part should be basically identical as they are based on electric circuit theory, the equations and algorithms may be different. Thus, in order to ensure the validity of all different techniques, comparative test calculations should be carried out. Section 4 provides such a test power grid model, which refers to the $400-\mathrm{kV}$ power system in Finland in the 1970s. The size of this system may be regarded as ideal for tests since the numbers of the stations and lines and the complexity are high enough, but unnecessary troubles due to very large matrices are avoided in the computations and analyses. In Section 4, I also present the values of the earthing GIC to (from) the Earth at every station and of the GIC flowing in the lines due to a uniform eastward or northward geoelectric field of $1 \mathrm{~V} / \mathrm{km}$.
Properties of the transfer matrix that couples the earthing GICs to the geovoltages are studied in Section 5 by both a theoretical analysis and a numerical calculation. By considering first an idealized linear grid with identical equidistant earthed nodes lying in a uniform geoelectric field and then a station of the Finnish $400-\mathrm{kV}$ power system, Pirjola (2005a) shows numerically that the calculated GIC at one site does not change much if, instead of a large network, only a smaller grid in the vicinity of the particular site is included in the model. The same topic is studied in Section 5 by considering the behavior of the elements of the transfer matrix.

In summary, this paper has three main objectives: to derive an explicit linear relationship between the geoelectric field and the earthing GICs in a power system (Section 2), to introduce a power grid model applicable to test computations of GICs (Section 4), and to consider properties of the transfer matrix between the geovoltages and the earthing GICs (Sections 3 and 5). The first and third objectives yield a new insight into the physical processes associated with GICs.

\section{Matrix Equations for GICs in a Power Grid}

Since geoelectromagnetic variations are slow compared, for example, to the 50- and $60-\mathrm{Hz}$ frequencies used in electric power transmission, a dc modeling of GICs is acceptable (at least as a first approximation). Figure 1 gives a schematic view of a network having $N$ discrete nodes, called stations, earthed by resistances $R_{i}^{\mathrm{e}}(i=1, \ldots, N)$. The resistance of a conductor line connecting stations $i$ and $m(i, m=1, \ldots, N)$ is denoted by $R_{i m}^{\mathrm{n}}$. Lehtinen and Pirjola (1985) derived the following formula for the $N \times$ 1 column matrix $\mathbf{I}_{\mathrm{e}}$, which consists of GICs, denoted by $I_{\mathrm{e}, m}$ ( $m=1, \ldots, N)$, and called the earthing GICs, to (from) the Earth (with the positive direction into the Earth) at the stations:

$$
\mathbf{I}_{\mathrm{e}}=\left(\mathbf{1}+\mathbf{Y}_{\mathrm{n}} \mathbf{Z}_{\mathrm{e}}\right)^{-1} \mathbf{J}_{\mathrm{e}}
$$

This equation, which has been presented in many other publications as well (e.g. Pirjola, 2005a, 2007, 2008a), provides a solution for the engineering part of a GIC calculation. The symbol 1 is an $N \times N$ unit identity matrix. The $N \times N$ earthing impedance matrix $\mathbf{Z}_{\mathrm{e}}$ and the $N \times N$ network admittance matrix $\mathbf{Y}_{\mathrm{n}}$, as well as the $N \times 1$ column matrix $\mathbf{J}_{\mathrm{e}}$, which includes the information about the geoelectric field, are defined below.

Multiplying $\mathbf{I}_{\mathrm{e}}$ by $\mathbf{Z}_{\mathrm{e}}$ gives the voltages between the earthing points and a remote earth associated with the flow of the currents $I_{\mathrm{e}, m}(m=1, \ldots, N)$. Including the voltages in an $N \times 1$ column matrix $\mathbf{U}_{\text {cur }}$, we thus have

$$
\mathbf{U}_{\text {cur }}=\mathbf{Z}_{\mathrm{e}} \mathbf{I}_{\mathrm{e}} \text {. }
$$

(Following the original notation by Lehtinen and Pirjola (1985), the subscript 'cur' indicates that voltages associated with earthing currents are considered.) By the reciprocity theorem, $\mathbf{Z}_{\mathrm{e}}$ is symmetric. If the stations are distant enough, thereby making the effect of one earthing current on the voltage at another station negligible, $\mathbf{Z}_{\mathrm{e}}$ is simply diagonal, with the elements equaling the earthing resistances $R_{i}^{\mathrm{e}}(i=1, \ldots, N)$. Equation (2) should thus be considered 


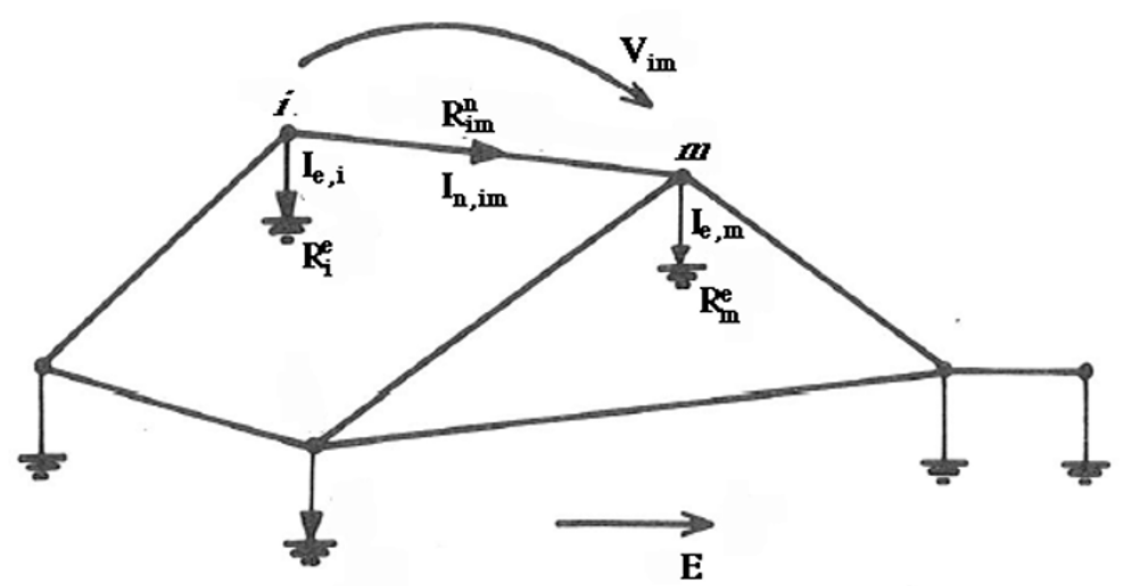

Fig. 1. Network consisting of $N$ nodes, called stations, earthed by resistances $R_{i}^{\mathrm{e}}(i=1, \ldots, N)$. The resistance of a conductor line between stations $i$ and $m(i, m=1, \ldots, N)$ is denoted by $R_{i m}^{\mathrm{n}}$. An external geoelectric field $\mathbf{E}$ produces geomagnetically induced currents (GICs) to (from) the Earth denoted by $I_{\mathrm{e}, i}$ and in the conductors denoted by $I_{\mathrm{n}, i m}$. The GICs are calculated by using the geovoltages $V_{i m}$ obtained by integrating $\mathbf{E}$ along the conductors. This figure is a slightly modified version of figure 1 by Lehtinen and Pirjola (1985).

as the definition of $\mathbf{Z}_{\mathrm{e}}$. The voltages included in $\mathbf{U}_{\text {cur }}$ are physically associated with the electric field that produces a part of the (telluric) currents driven by the geoelectric field flowing through the earthed network.

The matrix $\mathbf{Y}_{\mathrm{n}}$ is defined by

$$
(i \neq m): Y_{\mathrm{n}, i m}=-\frac{1}{R_{i m}^{\mathrm{n}}}, \quad(i=m): Y_{\mathrm{n}, i m}=\sum_{k=1, k \neq i}^{N} \frac{1}{R_{i k}^{\mathrm{n}}}
$$

which directly shows that $\mathbf{Y}_{\mathrm{n}}$ is a symmetric matrix. The elements $J_{\mathrm{e}, m}(m=1, \ldots, N)$ of the column matrix $\mathbf{J}_{\mathrm{e}}$ are

$$
J_{\mathrm{e}, m}=\sum_{i=1, i \neq m}^{N} \frac{V_{i m}}{R_{i m}^{\mathrm{n}}}
$$

The geovoltage $V_{i m}$ is produced by the horizontal geoelectric field $\mathbf{E}$, which is external from the viewpoint of the network in the engineering part of a GIC calculation. Thus, $V_{i m}$ is obtained by integrating $\mathbf{E}$ along the path defined by the conductor line from station $i$ to station $m(i, m=$ $1, \ldots, N)$ :

$$
V_{i m}=\int_{i}^{m} \mathbf{E} \cdot d \mathbf{s}
$$

Generally, the geoelectric field is rotational, which implies that the integral in Eq. (5) is path-dependent, and the integration route has to correspond to the conductor between $i$ and $m$ (Boteler and Pirjola, 1998). Equation (1) directly shows that, assuming perfect earthings ("pe"), i.e., $\mathbf{Z}_{\mathrm{e}}=0$, the GICs included in $\mathbf{I}_{\mathrm{e}}$ equal the elements of $\mathbf{J}_{\mathrm{e}}$, called the "pe" earthing currents.

Additional details associated with the derivation of Eqs. (1)-(5) are given by Lehtinen and Pirjola (1985). A formula for the GIC flowing in a conductor between stations $i$ and $m(i, m=1, \ldots, N)$, denoted by $I_{\mathrm{n}, i m}$, can also be derived (Lehtinen and Pirjola, 1985; Pirjola, 2007, 2008a), but this formula is less important in practice than Eq. (1) because the earthing GICs create problems by saturating power system transformers. Referring to the electrical circuit theory, we can say that the voltages $V_{i m}$ and those in $\mathbf{U}_{\text {cur }}$ correspond to the open-circuit electromotive force and the voltage drop in a battery, respectively, when the currents $I_{\mathrm{n}, i m}$ in the conductors are considered.

When applying Eqs. (1)-(5) to calculating GICs in a real three-phase power system earthed via transformer neutrals at stations, the three phases are usually treated as one circuit element. The resistance of this element is then one third of that of a single phase, and it carries a GIC threefold higher than the current in a single conductor. For convenience, the (total) earthing resistances of the stations are defined to include the actual earthing resistances, the transformer resistances, and the resistances of possible neutral point reactors (or any other resistors) in the earthing leads of transformer neutrals (all resistances in series). It should be noted that the technical reason for installing neutral point reactors is to decrease possible earth-fault currents, which is important for the safety of the power system. In terms of GICs, a neutral point reactor just provides an additional resistance in the earthing lead as a "spin-off". Mäkinen (1993) and Pirjola (2005a) describe the application of Eqs. (1)-(5) to a power grid in further detail.

Here, I use the Cartesian coordinate system standard in geoelectromagnetics with the $x y$ plane lying at the Earth's surface and the $x, y$, and $z$ axes pointing to the north, to the east, and downwards, respectively. The horizontal geoelectric field $\mathbf{E}=\left(E_{x}, E_{y}\right)$ is assumed to be known at $M$ grid points. In this study, we do not specify the details of the grid, except that its size and location have to enable the estimation of the electric field at all points of the network in which the GICs are investigated. The grid may be either regular or irregular, it can be rectangular or it may follow the latitudes and longitudes, and so on. For example, Wik et al. (2008) made a GIC calculation of the southern Swedish 400-kV power system using an 88-point grid (see their Fig. 2). The voltages $V_{i m}$ are assumed to be linear combinations of the values of the field components $E_{x, k}$ and $E_{y, k}$ at the grid points $(k=1, \ldots, M)$ :

$$
V_{i m}=\sum_{k=1}^{M}\left(\lambda_{k}^{i m} E_{x, k}+\eta_{k}^{i m} E_{y, k}\right)
$$




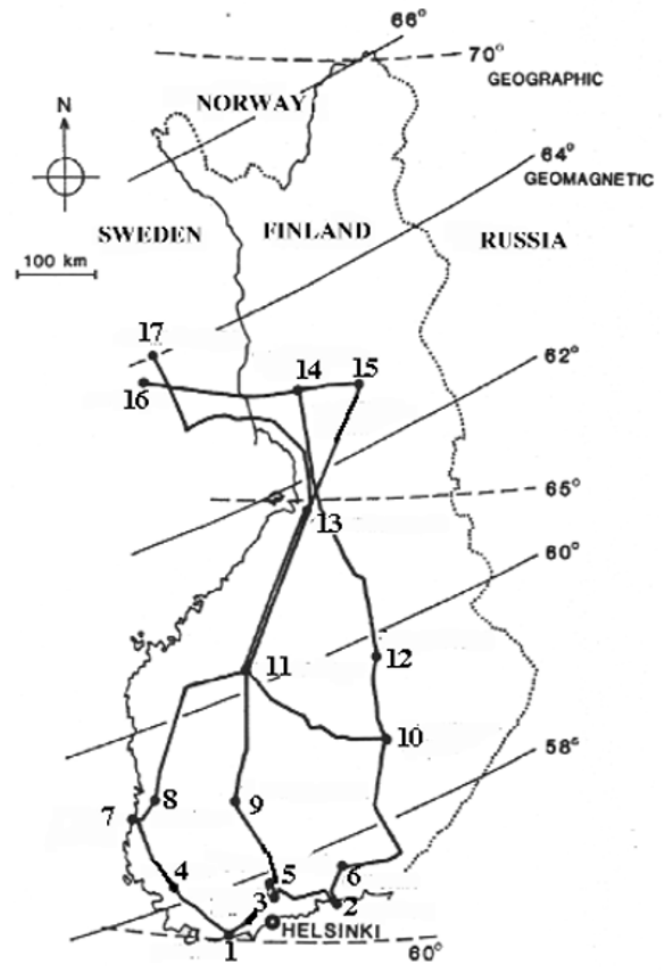

Fig. 2. Finnish 400-kV electric power transmission grid in its configuration valid in October 1978 to November 1979 (Pirjola and Lehtinen, 1985; Pirjola, 2005a). The names of the stations numbered from 1 to 17 are given in Table 1.

The coefficients $\lambda_{k}^{i m}$ and $\eta_{k}^{i m}$ have the dimension of length. They depend on the numerical method of computing the integral in Eq. (5), which involves both the interpolation from the grid points $k$ to $x, y$ values lying at the conductor and the numerical integration itself. It is clear that the coefficients $\lambda_{k}^{i m}$ and $\eta_{k}^{i m}$ decrease with increasing distance from the grid point $k$ to the conductor connecting stations $i$ and $m$. Different techniques exist for the interpolation. The only implicit assumption included in Eq. (6) is that the linear dependence on the geoelectric field components is maintained (which is a very natural requirement). In this paper, however, I will not go into the details of the numerical computations of $\lambda_{k}^{i m}$ and $\eta_{k}^{i m}$ because the purpose of presenting Eq. (6) is to derive a simple linear relationship between the geoelectric field and the GICs rather than to provide detailed instructions and advice for numerical calculation techniques of $\lambda_{k}^{i m}$ and $\eta_{k}^{i m}$. Moreover, the examples of the calculation in Sections 4 and 5 refer to Eqs. (1)-(5) under a uniform geoelectric field.

Substituting Eq. (6) into Eq. (4) gives

$$
J_{\mathrm{e}, m}=\sum_{k=1}^{M}\left(E_{x, k} \sum_{i=1, i \neq m}^{N} \frac{\lambda_{k}^{i m}}{R_{i m}^{\mathrm{n}}}+E_{y, k} \sum_{i=1, i \neq m}^{N} \frac{\eta_{k}^{i m}}{R_{i m}^{\mathrm{n}}}\right)
$$

Let us define $N \times M$ matrices as $\boldsymbol{\alpha}$ and $\boldsymbol{\beta}$ so that their elements are

$$
\begin{array}{r}
\alpha_{m k}=\sum_{i=1, i \neq m}^{N} \frac{\lambda_{k}^{i m}}{R_{i m}^{\mathrm{n}}}, \quad \beta_{m k}=\sum_{i=1, i \neq m}^{N} \frac{\eta_{k}^{i m}}{R_{i m}^{\mathrm{n}}} \\
(m=1, \ldots, N ; k=1, \ldots, M)
\end{array}
$$

Using Eqs. (7) and (8), the matrix $\mathbf{J}_{\mathrm{e}}$ can be expressed as the sum of two matrix products in the form

$$
\mathbf{J}_{\mathrm{e}}=\boldsymbol{\alpha} \mathbf{E}_{x}+\boldsymbol{\beta} \mathbf{E}_{y}
$$

where $\mathbf{E}_{x}$ and $\mathbf{E}_{y}$ are $M \times 1$ matrices giving the geoelectric components $E_{x, k}$ and $E_{y, k}$ at the grid points. We combine the $\boldsymbol{\alpha}$ and $\boldsymbol{\beta}$ matrices to be one single $N \times 2 M$ matrix $\mathbf{A}$ so that $A_{i j}=\alpha_{i j}$ for $j=1, \ldots, M$, and $A_{i j}=\beta_{i j-M}$ for $j=M+1, \ldots, 2 M$. Similarly, the $2 M \times 1$ matrix $\mathbf{E}$ is a combination of $\mathbf{E}_{x}$ and $\mathbf{E}_{y}$, such that $E_{k}=E_{x, k}$ for $k=1, \ldots, M$ and $E_{k}=E_{y, k-M}$ for $k=M+1, \ldots, 2 M$. Equation (9) is then simplified to be

$$
\mathbf{J}_{\mathrm{e}}=\mathbf{A E}
$$

From Eqs. (1) and (10), I then obtain

$$
\mathbf{I}_{\mathrm{e}}=\mathbf{G E}
$$

where the $N \times 2 M$ matrix $\left(\mathbf{1}+\mathbf{Y}_{\mathrm{n}} \mathbf{Z}_{\mathrm{e}}\right)^{-1} \mathbf{A}$ is denoted by G. The line and earthing resistances of the power grid are included in the matrices $\mathbf{Y}_{\mathrm{n}}$ and $\mathbf{Z}_{\mathrm{e}}$. The matrix $\mathbf{A}$ characterizes the geometry of the network and the numerical computation of the voltages in Eq. (5), and it is also affected by the line resistances via Eq. (8). Separating the influences of the different factors by analyzing the matrix $\mathbf{G}$ is obviously a challenge in practice.

Although the time $t$ is not explicitly included in the above discussion, the matrices $\mathbf{E}=\mathbf{E}(t)$ and $\mathbf{I}_{\mathrm{e}}=\mathbf{I}_{\mathrm{e}}(t)$ are functions of time. The matrix $\mathbf{G}$ does not depend on time (except for the fact that it varies with possible changes in the power system configuration or resistances, but it is a different issue). Considering $L$ time moments, we may still use Eq. (11), but $\mathbf{I}_{\mathrm{e}}$ is an $N \times L$ matrix and $\mathbf{E}$ is a $2 M \times$ $L$ matrix, with the columns corresponding to consecutive times. Let us then denote a single row of the matrix $\mathbf{I}_{\mathrm{e}}$ by $\mathbf{g}$ and the corresponding row of the matrix $\mathbf{G}$ by $\mathbf{W}$. We thus refer to a particular station of the network, for example, one equipped with GIC measurements. The rows $\mathbf{g}$ and $\mathbf{W}$ are 1 $\times L$ and $1 \times 2 M$ matrices, respectively, and

$$
\mathbf{g}=\mathbf{W E}
$$

Time series of measured GIC data and of calculated geoelectric field values combined with Eq. (12) can enable the determination of $\mathbf{W}$. For example, a least-square fitting can be used, which, as well as the possibility of applying neural network techniques, has preliminarily been considered in the context of studies of GIC in the southern Swedish 400$\mathrm{kV}$ power grid (private communication). Using these techniques, we can avoid the power system modeling presented by Eq. (1)-(5) and included implicitly in $\mathbf{W}$ and, instead, an experimental relation would be obtained between GICs and geoelectric data. Details of such investigations are, however, beyond the scope of this paper. It is important to note that $\mathbf{W}$ depends on the power grid configuration and a set of resistances - and is not valid for other power grid configurations and sets of resistances. Therefore, the $\mathbf{W}$ should be updated after every change in the power system. 


\section{Effect of Changing the Value of an Earthing Resistance on GICs at a Station}

Let $a$ and $b$ denote a GIC at a site in a power system produced by a uniform northward or a uniform eastward geoelectric field of $1 \mathrm{~V} / \mathrm{km}$, respectively. Then, due to linearity, a GIC created by any uniform horizontal geoelectric field can be expressed as

$$
\operatorname{GIC}(t)=a E_{x}(t)+b E_{y}(t)
$$

where the north and east components of the field, as well as GIC, are regarded as being dependent on the time $t$. A unit of $a$ and $b$ is expressed as amperes times kilometer per volt $(\mathrm{A} \mathrm{km} / \mathrm{V})$, when $E_{x}$ and $E_{y}$ are expressed in volts per kilometer $(\mathrm{V} / \mathrm{km})$ and the GIC is expressed in amperes. Considering separate time moments $t=t_{1}, \ldots, t_{L}$, Eq. (13) is actually a special case of Eq. (12) with $M=1$.

Equation (13) can also be applied to an experimental determination of the coefficients $a$ and $b$ by using measured GIC data together with geoelectric field values computed from geomagnetic recordings (compare the end of Section 2; see, for example, Wik et al., 2008). The values of $a$ and $b$ obtained by measurements are often different from the values by a model calculation performed with a uniform field of $1 \mathrm{~V} / \mathrm{km}$ because the geoelectric field significantly depends on the Earth's conductivity. However, the ratio $a / b$ is expected to be the same for both the experimental and theoretically calculated coefficients. The ratio indicates the relative importance of the two geoelectric components for the GIC at the considered site and depends on the grid configuration and resistances (see, for example, Wik et al., 2008). In practical situations, the calculated geoelectric field amplitudes may be scaled to incorrect values due to lack of the precise ground conductivity data, but the two geoelectric components should obtain the correct relative weight when the calculated values are fitted to the measured GIC data.

Based on Eq. (1), I now investigate the effect of the (total) earthing resistance $R_{j}^{\mathrm{e}}$ of a given station $j(j=1, \ldots, N)$ on the ratio $a / b$ associated with the earthing GIC, $I_{\mathrm{e}, j}$ at the same station. In practice, such a study may be useful when we cannot obtain reliable information on an additional resistor in the earthing lead of a transformer neutral during GIC measurements. The diagonal elements $Z_{\mathrm{e}, i i}$ of the earthing impedance matrix equal the resistances $R_{i}^{\mathrm{e}}(i=1, \ldots, N)$. As mentioned in Section 2, the total earthing resistances of the stations include the actual earthing resistances, the transformer resistances, and the resistances of any resistors in the earthing leads of transformer neutrals. Based on the definition of $\mathbf{Z}_{\mathrm{e}}$, it is clear that the off-diagonal elements of $\mathbf{Z}_{\mathrm{e}}$ are associated with the actual earthing resistances of the stations, which implies some coupling with the diagonal elements as well. However, we now assume that possible changes in the (total) earthing resistance $R_{j}^{\mathrm{e}}$ considered do not refer to the actual earthing resistance, which agrees with thinking of an installation or a removal of a resistor in the neutral lead. Consequently, the only quantity affected by the change and included in the GIC calculation is the element $Z_{\mathrm{e}, j j}\left(=R_{j}^{\mathrm{e}}\right)$.
The elements of the matrix $\mathbf{1}+\mathbf{Y}_{\mathrm{n}} \mathbf{Z}_{\mathrm{e}}$, denoted by $\mathbf{D}$, are

$$
D_{m p}=\delta_{m p}+\sum_{s=1}^{N} Y_{\mathrm{n}, m s} Z_{\mathrm{e}, s p} \quad(m, p=1, \ldots, N)
$$

where the symbol $\delta_{m p}$ stands for the Kronecker delta (= 1 if $m=p$, and $=0$ if $m \neq p$ ). Equation (14) shows that $Z_{\mathrm{e}, j j}$ only affects the elements of $\mathbf{D}$ that lie in the $j$ th column, i.e. $p=j$. (In fact, all elements $Z_{\mathrm{e}, r j}(r=$ $1, \ldots, N)$ only directly contribute to the $j$ th column of D but because $Z_{\mathrm{e}, r j}=Z_{\mathrm{e}, j r}$ indirect effects also appear elsewhere.) Consequently, the cofactors of the elements in the $j$ th column of $\mathbf{D}$ do not depend on $Z_{\mathrm{e}, j j}$. From the elementary matrix calculus, we know that the inverse of $\mathbf{D}$, needed in Eq. (1), is obtained by transposing the matrix composed of the cofactors of $\mathbf{D}$ and by dividing by the determinant of $\mathbf{D}(=\operatorname{det}(\mathbf{D}))$. Thus, $Z_{\mathrm{e}, j j}$ contributes to the elements in the $j$ th row of $\mathbf{D}^{-1}$ only through a common denominator $\operatorname{det}(\mathbf{D})$.

Equation (1) gives that

$$
I_{\mathrm{e}, j}=\sum_{i=1}^{N}\left(\mathbf{D}^{-1}\right)_{j i} J_{\mathrm{e}, i}
$$

By using the above-mentioned facts,

$$
I_{\mathrm{e}, j}=\frac{1}{\operatorname{det}(\mathbf{D})} \sum_{i=1}^{N} \psi_{j i} J_{\mathrm{e}, i}
$$

where $\psi_{j i}(j, i=1, \ldots, N)$ is an element of the transposed matrix of the cofactors of $\mathbf{D}$ denoted by $[\operatorname{cof}(\mathbf{D})]^{*}$. (The asterisk means transposing.) It is important that the elements $\psi_{j i}$ included in Eq. (16) are not affected by $Z_{\mathrm{e}, j j}\left(=R_{j}^{\mathrm{e}}\right)$. According to Eqs. (4) and (5), the column matrix $\mathbf{J}_{\mathrm{e}}$ is independent of all earthing resistances, including $R_{j}^{\mathrm{e}}$. Consequently, the effects of possible changes of $R_{j}^{\mathrm{e}}$ on $I_{\mathrm{e}, j}$, i.e., on the earthing GIC at the site $j$ considered, only come by the term $\operatorname{det}(\mathbf{D})$ in Eq. (16). The elements $J_{\mathrm{e}, i}(i=1, \ldots, N)$ introduce the information on the geoelectric field.

It thus follows that if $I_{\mathrm{e}, j}$ is calculated in two different geoelectric field situations, expressed by $J_{\mathrm{e}, i}(1)$ and $J_{\mathrm{e}, i}(2)$ $(i=1, \ldots, N)$, and the results are $I_{\mathrm{e}, j}(1)$ and $I_{\mathrm{e}, j}(2)$, the ratio $I_{\mathrm{e}, j}(1) / I_{\mathrm{e}, j}(2)$ is not affected by changes in $R_{j}^{\mathrm{e}}$. For example, we obtain $a=I_{j}(1)$ and $b=I_{j}(2)$ for a uniform northward and eastward geoelectric field of $1 \mathrm{~V} / \mathrm{km}$, as discussed above. Thus, the ratio $a / b$ at each station is independent of the installation or removal of an additional resistor in the neutral point of the transformer.

\section{Test Model for GIC Calculations}

Although the solution of the engineering part in the calculation of GICs in a power system is based on fundamental circuit theory, including Ohm's and Kirchhoff's laws, the final algorithms and programs to be used may be different. This could be due to the inclusion of the transformer resistances in the (total) earthing resistances of the stations, as is done in this paper, to differences in the treatments of autotransformers, at which two different voltage levels are in a galvanic connection, thereby enabling the flow of GICs from one level to another, or to any other differences. 
Table 1. Stations of the Finnish 400-kV power grid shown in Fig. 2. Their Cartesian east and north coordinates and their (total) earthing resistances are also given (Pirjola and Lehtinen, 1985).

$$
\text { Coordinates }[\mathrm{km}]
$$

\begin{tabular}{|c|c|c|c|}
\hline$\underline{\text { Station }}$ & $\underline{\text { East }}$ & North & Earthing Resistance $[\Omega]$ \\
\hline $1=$ Inkoo & 351.44 & 58.17 & 0.43 \\
\hline $2=$ Loviisa & 492.79 & 97.60 & 0.36 \\
\hline $3=$ Nurmijärvi & 411.06 & 106.25 & 0.66 \\
\hline $4=$ Lieto & 282.69 & 120.19 & 0.60 \\
\hline $5=$ Hyvinkää & 404.33 & 125.00 & 0.47 \\
\hline $6=$ Koria & 499.52 & 146.63 & 0.45 \\
\hline $7=$ Olkiluoto & 227.40 & 206.73 & 0.33 \\
\hline $8=$ Ulvila & 258.65 & 230.29 & 0.64 \\
\hline 9= Kangasala & 361.06 & 228.85 & 0.86 \\
\hline $10=$ Huutokoski & 556.25 & 305.29 & 0.57 \\
\hline 11 = Alajärvi & 375.00 & 394.23 & 0.98 \\
\hline $12=$ Alapitkä & 543.27 & 409.13 & 1.14 \\
\hline $13=$ Pikkarala & 452.88 & 593.75 & 0.70 \\
\hline 14 = Petäjäskoski & 441.83 & 746.15 & 0.47 \\
\hline $15=$ Pirttikoski & 520.19 & 752.40 & 0.95 \\
\hline $16=$ Letsi & 244.71 & 755.77 & 0 \\
\hline $17=$ Messaure & 256.73 & 789.90 & 0 \\
\hline
\end{tabular}

For example, the Mesh Impedance Matrix Method (MIMM) and the Network Admittance Matrix Method (NAMM), both of which also refer to load-flow calculation techniques traditionally used by electric power industry, are applicable to GIC computations (Boteler and Pirjola, 2004). NAMM can be shown to be equivalent with the method developed independently by Lehtinen and Pirjola (1985), called LP and summarized in Section 2. In fact, LP is slightly more general as it allows the possibility of non-zero off-diagonal elements in the earthing impedance matrix. The computations and formulas discussed in this paper are based on LP.

For the comparisons, it is necessary to apply different GIC computation methods to the same power grid model. Figure 2, which refers to the Finnish 400-kV system in October 1978 to November 1979 (Pirjola and Lehtinen, 1985; Pirjola, 2005a), is a recommendable choice for such a test model because the numbers of stations and transmission lines are high enough (17 stations and 19 transmission lines) but it is not too large to unnecessarily complicate the computations and analyses. (Note that between stations 11 and 13 there are actually two transmission lines but that in the GIC studies these are treated as a single line.) The earthing resistances of the stations and the resistances of the transmission lines are given in Tables 1 and 2, respectively. These data have also been presented by Pirjola and Lehtinen (1985). As indicated in Section 2, the three phases are considered to be one circuit element, and the earthing resistances include the effects of both the station earthings and the transformers. No neutral point reactors (or other resistors) in the earthing leads are assumed. This is reasonable because most of the reactors were installed in the Finnish $400-\mathrm{kV}$ system later than the period referred to in Fig. 2; as such, the test model remains simpler. Similar to Pirjola
Table 2. Lines and their resistances between stations A and B of the Finnish 400-kV power grid shown in Fig. 2 (Pirjola and Lehtinen, 1985). Station A Station B Line Resistance [ $[\Omega]$

\begin{tabular}{|c|c|c|}
\hline $1=$ Inkoo & $4=$ Lieto & 0.59 \\
\hline $1=$ Inkoo & $5=$ Hyvinkää & 0.65 \\
\hline $2=$ Loviisa & $3=$ Nurmijärvi & 0.58 \\
\hline $2=$ Loviisa & $6=$ Koria & 0.33 \\
\hline $3=$ Nurmijärvi & $5=$ Hyvinkää & 0.076 \\
\hline $4=$ Lieto & $7=$ Olkiluoto & 0.59 \\
\hline $5=$ Hyvinkää & $9=$ Kangasala & 1.04 \\
\hline $6=$ Koria & $10=$ Huutokoski & 1.36 \\
\hline $7=$ Olkiluoto & $8=$ Ulvila & 0.27 \\
\hline $8=$ Ulvila & 11 = Alajärvi & 1.98 \\
\hline 9 = Kangasala & 11 = Alajärvi & 1.47 \\
\hline $10=$ Huutokoski & 11 = Alajärvi & 1.88 \\
\hline $10=$ Huutokoski & $12=$ Alapitkä & 0.98 \\
\hline 11 = Alajärvi & $13=$ Pikkarala & 0.96 \\
\hline 12 = Alapitkä & $14=$ Petäjäskoski & 3.20 \\
\hline $13=$ Pikkarala & $15=$ Pirttikoski & 1.46 \\
\hline $13=$ Pikkarala & $17=$ Messaure & 2.89 \\
\hline 14 = Petäjäskoski & $15=$ Pirttikoski & 0.71 \\
\hline 14 = Petäjäskoski & $16=$ Letsi & 1.70 \\
\hline
\end{tabular}

and Lehtinen (1985), the earthing resistances of stations 16 and 17 are set equal to zero to approximately account for the connection to the Swedish $400-\mathrm{kV}$ network. Thus, the currents $I_{\mathrm{e}, 16}$ and $I_{\mathrm{e}, 17}$ are actually not earthing GICs but include currents that flow between the Finnish and Swedish systems.

Table 1 also gives the east and north coordinates of the stations in kilometers in a Cartesian coordinate system. (The origin located southwest of Finland does not play any role because only the relative positions of different parts and sites of the power grid are important.) The coordinates have been simply measured from a map and, therefore, possibly contain small inaccuracies. The resistance values presented in Tables 1 and 2 are based on information received from the power company in the beginning of the 1980s when GIC model calculations were initiated in Finland. In particular, the resistance data given in Table 1 may include approximations. The power grid model provided by Fig. 2 and Tables 1 and 2 is sufficient for GIC calculation tests, although the data of the model are slightly different from those of the old Finnish 400-kV system.

Based on the formulas given in Section 2 and the coordinate and resistance data expressed in Tables 1 and 2, Table 3 gives GICs that flow to (positive) or from (negative) the Earth at the stations of the grid considered under a uniform eastward and a uniform northward geoelectric field of $1 \mathrm{~V} / \mathrm{km}$. Table 4 presents GICs in the transmission lines (as positive from station A to station B). The GICs in these lines are calculated using the formula given, for example, by Pirjola (2008a) (see Section 2). In the present calculation, I assumed that the earthing impedance matrix $\mathbf{Z}_{\mathrm{e}}$ is diagonal. (Naturally, a more general test model should require the possibility of non-zero off-diagonal elements of 
Table 3. GICs at the stations of the Finnish $400-\mathrm{kV}$ power grid shown in Fig. 2 under northward and eastward 1-V/km uniform horizontal geoelectric fields. GICs flowing to (from) the Earth are defined as positive (negative).

\begin{tabular}{|c|c|c|}
\hline \multirow[t]{3}{*}{ Station } & \multicolumn{2}{|c|}{$\underline{G I C}[A]$} \\
\hline & \multicolumn{2}{|c|}{ Electric field $1 \mathrm{~V} / \mathrm{km}$} \\
\hline & Eastward & Northward \\
\hline $1=$ Inkoo & 4.1 & -107.5 \\
\hline $2=$ Loviisa & 58.0 & -88.6 \\
\hline $3=$ Nurmijärvi & -1.1 & -35.7 \\
\hline $4=$ Lieto & -25.4 & -36.8 \\
\hline $5=$ Hyvinkää & 0.7 & -16.2 \\
\hline $6=$ Koria & 28.0 & -26.4 \\
\hline $7=$ Olkiluoto & -102.5 & 14.9 \\
\hline $8=$ Ulvila & -21.0 & 8.2 \\
\hline $9=$ Kangasala & -26.2 & -4.0 \\
\hline $10=$ Huutokoski & 79.5 & -15.4 \\
\hline 11 = Alajärvi & -18.6 & 15.3 \\
\hline $12=$ Alapitkä & 27.8 & 1.6 \\
\hline $13=$ Pikkarala & 52.5 & 36.1 \\
\hline $14=$ Petäjäskoski & 36.4 & 84.8 \\
\hline $15=$ Pirttikoski & 68.8 & 63.9 \\
\hline $16=$ Letsi & -105.9 & 29.1 \\
\hline $17=$ Messaure & -55.2 & 76.6 \\
\hline
\end{tabular}

Table 4. GICs flowing between stations A and B (as positive from A to B) in the lines of the Finnish 400-kV power grid shown in Fig. 2 under northward and eastward 1-V/km uniform horizontal geoelectric fields. Station A Station B GIC [A]

\begin{tabular}{|c|c|c|c|}
\hline & & \multicolumn{2}{|c|}{ Flectric field $1 \mathrm{~V} / \mathrm{km}$} \\
\hline & & Eastward & Northward \\
\hline $1=$ Inkoo & $4=$ Lieto & -87.7 & 64.2 \\
\hline $1=$ Inkoo & $5=$ Hyvinkää & 83.6 & 43.4 \\
\hline $2=$ Loviisa & $3=$ Nurmijärvi & -103.6 & 0.6 \\
\hline $2=$ Loviisa & $6=$ Koria & 45.6 & 88.0 \\
\hline $3=$ Nurmijärvi & $5=$ Hyvinkää & -102.5 & 36.3 \\
\hline $4=$ Lieto & $7=$ Olkiluoto & -62.3 & 100.9 \\
\hline $5=$ Hyvinkää & $9=$ Kangasala & -19.6 & 95.9 \\
\hline $6=$ Koria & $10=$ Huutokoski & 17.6 & 114.4 \\
\hline $7=$ Olkiluoto & $8=$ Ulvila & 40.2 & 86.1 \\
\hline $8=$ Ulvila & 11 = Alajärvi & 61.2 & 77.9 \\
\hline $9=$ Kangasala & 11 = Alajärvi & 6.6 & 99.9 \\
\hline $10=$ Huutokoski & 11 = Alajärrvi & -62.6 & 34.7 \\
\hline $10=$ Huutokoski & 12 = Alapitkä & 0.7 & 95.1 \\
\hline 11 = Alajärvi & $13=$ Pikkarala & 23.8 & 197.1 \\
\hline 12 = Alapitkä & $14=$ Petäjäskoski & -27.1 & 93.4 \\
\hline 13 = Pikkarala & $15=$ Pirttikoski & 26.5 & 84.4 \\
\hline $13=$ Pikkarala & $17=$ Messaure & -55.2 & 76.6 \\
\hline 14 = Petäjäskoski & $15=$ Pirttikoski & 42.4 & -20.5 \\
\hline 14 = Petäjäskoski & $16=$ Letsi & -105.9 & 29.1 \\
\hline
\end{tabular}

$\mathbf{Z}_{\mathrm{e}}$, too, but such a complication is neglected now.)

Tables 3 and 4 show that GICs in the lines tend, on average, to exceed those through transformers. The means of the absolute values of the GICs in Table 3 are 41.9 A (eastward) and 38.9 A (northward), and the corresponding values for Table 4 are 51.3 A (eastward) and 75.7 A (northward). This result is in agreement with the theoretical calculation using an ideal system and the numerical computation using the Finnish 400-kV grid (Pirjola, 2005b). The GICs flowing through several earthings concentrate in lines in the middle of the network. As a result of this, the average GICs in the lines exceed the average earthing GICs. A comparison of Tables 3 and 4 with the GIC results given by Pirjola and Lehtinen (1985) reveals small differences. This is due to the fact that the calculations discussed by Pirjola and Lehtinen (1985) were based on another earlier measurement of the station coordinates from a map, and there are minor deviations. Although the GIC magnitudes in Tables 3 and 4 vary largely from site to site, the same order of GICs was measured in the Finnish high-voltage system during geomagnetic storms (Elovaara et al., 1992). This provides an indirect verification of the validity of the calculations, although an exact agreement with a space storm event cannot be presumed because real geoelectric fields are not precisely uniform and equal to $1 \mathrm{~V} / \mathrm{km}$ northwards or eastwards.

\section{Properties of the Transfer Matrix}

Equations (1) and (4) express the GIC flowing through transformers to (from) the Earth in terms of the voltages associated with the horizontal geoelectric field along the transmission lines expressed by Eq. (5). The matrix formulas (11) and (12) give a direct relation between GICs and the geoelectric field. Although these formulas look simple, their interpretation is complicated because the transfer matrices $\mathbf{G}$ and $\mathbf{W}$ include the network parameters as well as the integration of the geoelectric field.

Let us now consider in greater detail the $N \times N$ matrix $\left(\mathbf{1}+\mathbf{Y}_{\mathrm{n}} \mathbf{Z}_{\mathrm{e}}\right)^{-1}$ appearing in Eq. (1) and denoted by $\mathbf{C}$. According to formulas (1) and (4), C plays the role of a transfer matrix between the geovoltages and the earthing GICs. Perfect earthings (i.e., $\mathbf{Z}_{\mathrm{e}}=0$ ), make $\mathbf{C}$ the identity matrix so that the earthing GICs $\mathbf{I}_{e}$ equal the "pe" currents $\mathbf{J}_{\mathrm{e}}$ (see Section 2). Lehtinen and Pirjola (1985) also give another interpretation of the equations derived for the currents produced by the geoelectric field in the earthings and the conductor lines by replacing the geoelectric field with an external injection of the "pe" currents $J_{\mathrm{e}, i}$ into the nodes $(i=1, \ldots, N)$. Although it is not said explicitly, this formulation clearly corresponds to the use of Norton's equivalent current sources instead of geovoltages in the transmission lines (see also Boteler and Pirjola, 2004). The external injection results in a correct formula (1) for the earthing GICs, but the "pe" line GICs $\left(=V_{i m} / R_{i m}^{\mathrm{n}} ; i\right.$, $m=1, \ldots, N)$ have to be added to the currents resulting from the injection. Consequently, if we do not think about an external injection but feed the "pe" currents $V_{i m} / R_{i m}^{\mathrm{n}}$ along the existing lines, no special addition is needed any more.

Based on Eq. (4) and on the relations $V_{i m}=-V_{m i}$ and $R_{i m}^{\mathrm{n}}=R_{m i}^{\mathrm{n}}$, we easily obtain that the sum of the "pe" currents $J_{\mathrm{e}, i}(i=1, \ldots, N)$ is zero. (It should be noted that the sum of the earthing GICs $I_{\mathrm{e}, m}(m=1, \ldots, N)$ is zero as well.) The external injections described by Lehtinen and Pirjola (1985) thus mean that an equal amount of current 
Table 5. Transfer matrix $\mathbf{C}=\left(\mathbf{1}+\mathbf{Y}_{\mathrm{n}} \mathbf{Z}_{\mathrm{e}}\right)^{-1}$ included in Eqs. (1) and (17) for the Finnish 400-kV power grid shown in Fig. 2. To make the table smaller, the last two columns (=16 and 17) are not shown. Their only non-zero elements, equal to one, are on the diagonal. The row and column numbers are also shown.

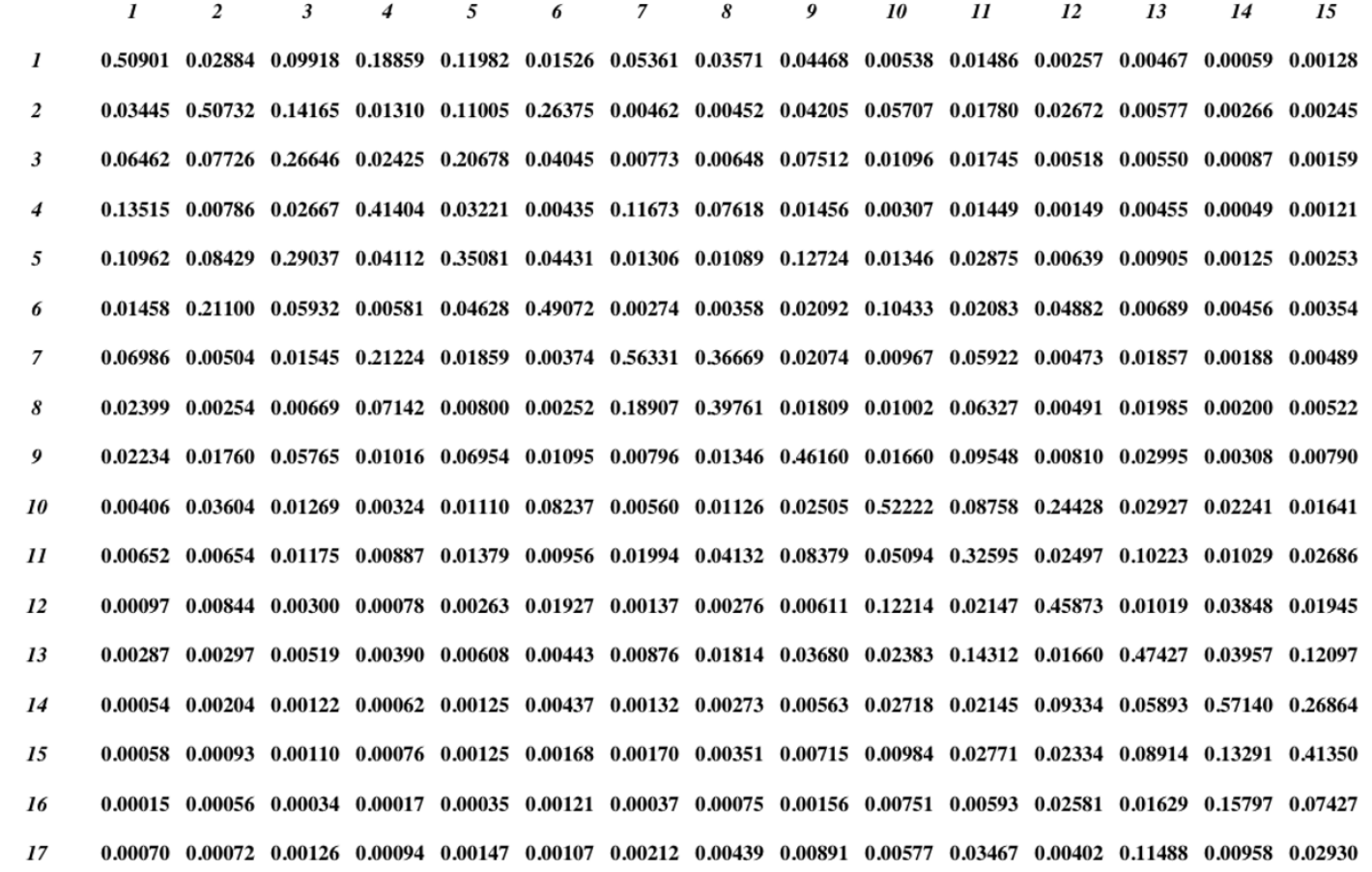

flows into and out of the network. However, the injections at the nodes are obviously independent of each other, which means that the current fed into (from) a single station flows in the form of earthing GICs $I_{\mathrm{e}, m}(m=1, \ldots, N)$ to (from) the Earth. Taking into account the equation

$$
\mathbf{I}_{\mathrm{e}}=\left(\mathbf{1}+\mathbf{Y}_{\mathrm{n}} \mathbf{Z}_{\mathrm{e}}\right)^{-1} \mathbf{J}_{\mathrm{e}}=\mathbf{C} \mathbf{J}_{\mathrm{e}}
$$

it can be seen that the part of an injected current $J_{\mathrm{e}, i}$ that is associated with the earthing GIC $I_{\mathrm{e}, m}$ is $C_{m i} J_{\mathrm{e}, i}(i, m=$ $1, \ldots, N)$. Consequently,

$$
J_{\mathrm{e}, i}=\sum_{m=1}^{N} C_{m i} J_{\mathrm{e}, i} \Rightarrow \sum_{m=1}^{N} C_{m i}=1
$$

i.e., the sum of the elements in every column of $\mathbf{C}$ is equal to one. This can be easily verified numerically by considering, for example, the Finnish $400-\mathrm{kV}$ grid introduced in Section 4, for which $N=17$. The matrix $\mathbf{C}$ for this special case is presented in Table 5 (without showing the last two columns). It should be emphasized that although the earthing impedance matrix $\mathbf{Z}_{\mathrm{e}}$ is assumed to be diagonal in the present numerical calculations for the Finnish $400-\mathrm{kV}$ system, the result on the sum of the column elements in $\mathbf{C}$ is always true because the theoretical derivation of Eq. (18) does not include any assumptions of $\mathbf{Z}_{\mathrm{e}}$.

As in Section 3, the matrix $\mathbf{1}+\mathbf{Y}_{\mathrm{n}} \mathbf{Z}_{\mathrm{e}}$ is denoted by $\mathbf{D}$, i.e., $\mathbf{C}=\mathbf{D}^{-1}$. Thus, referring to Eq. (16), we can write

$$
\mathbf{C}=\frac{1}{\operatorname{det}(\mathbf{D})}[\operatorname{cof}(\mathbf{D})]^{*}
$$

As, similarly to Section 3, the elements of $[\operatorname{cof}(\mathbf{D})]^{*}$ are denoted by $\psi_{j k}(j, k=1, \ldots, N)$, Eqs. (18) and (19) give

$$
\frac{1}{\operatorname{det}(\mathbf{D})} \sum_{m=1}^{N} \psi_{m i}=1
$$

for all values of $i=1, \ldots, N$. By the definition of a determinant,

$$
\operatorname{det}(\mathbf{D})=\sum_{m=1}^{N} D_{k m} \psi_{m k}
$$

for all values of $k=1, \ldots, N$. Equations (20) and (21) result in a special property of the matrix $D=\mathbf{1}+\mathbf{Y}_{\mathrm{n}} \mathbf{Z}_{\mathrm{e}}$, which for $i=k$ can be written as

$$
\sum_{m=1}^{N}\left(1-D_{i m}\right) \psi_{m i}=0
$$

for all values of $i=1, \ldots, N$. Finally, it is worth noting that although $\mathbf{1}, \mathbf{Y}_{\mathrm{n}}$, and $\mathbf{Z}_{\mathrm{e}}$ are symmetric, the matrices $\mathbf{C}$ and $\mathbf{D}$ need not be symmetric (see Table 5). Thus, for example, the sums of the elements in the rows of $\mathbf{C}$ generally differ from one.

The matrix $\mathbf{C}$ for the Finnish $400-\mathrm{kV}$ power system can now be considered. Table 5 shows that on each row of C the largest element is on the diagonal. When the GIC process is described by the injection of the "pe" currents $\mathbf{J}_{\mathrm{e}}$, each earthing GIC $I_{\mathrm{e}, m}(m=1, \ldots, 17)$ gets its greatest contribution from the injection at this same station $m$, which would appear to be a natural result. It is also seen that the largest element in every column is found on the diagonal, with the exception of the third column in which the element $(5,3)$ is the largest (i.e., slightly larger than the diagonal element $(3,3))$. Thus, a large part of an injected current 
Table 6. Elements $C(10, j)(j=1, \ldots, 17)$ of the tenth row of the transfer matrix $\mathbf{C}=\left(\mathbf{1}+\mathbf{Y}_{\mathrm{n}} \mathbf{Z}_{\mathrm{e}}\right)^{-1}$ included in Eqs. (1) and (17) for the Finnish 400-kV power grid shown in Fig. 2. The elements are arranged in order of value. The distance is given a distance between station 10 and station $j$. The neighborhood number is the number of stations from station 10 to station $j$ : it is 1 for the nearest stations, 2 for the second nearest stations, and so on.

\begin{tabular}{|c|c|c|c|}
\hline$C(10, j)$ & $j$ & Distance $[\mathrm{km}]$ & Neighborhood Number \\
\hline 0.00000 & 16 & 547.7 & 3 \\
\hline 0.00000 & 17 & 569.7 & 3 \\
\hline 0.00324 & 4 & 330.3 & 4 \\
\hline 0.00406 & 1 & 321.0 & 4 \\
\hline 0.00560 & 7 & 343.3 & 3 \\
\hline 0.01110 & 5 & 235.8 & 3 \\
\hline 0.01126 & 8 & 306.9 & 2 \\
\hline 0.01269 & 3 & 246.4 & 3 \\
\hline 0.01641 & 15 & 448.6 & 3 \\
\hline 0.02241 & 14 & 455.5 & 2 \\
\hline 0.02505 & 9 & 209.6 & 2 \\
\hline 0.02927 & 13 & 306.4 & 2 \\
\hline 0.03604 & 2 & 217.2 & 2 \\
\hline 0.08237 & 6 & 168.5 & 1 \\
\hline 0.08758 & 11 & 201.9 & 1 \\
\hline 0.24428 & 12 & 104.6 & 1 \\
\hline 0.52222 & 10 & $\mathbf{0}$ & $\mathbf{0}$ \\
\hline
\end{tabular}

$J_{\mathrm{e}, i}(i=1, \ldots, 17)$ flows into (from) the Earth at this same station $i$. The exception can be explained by the facts that stations 3 and 5 lie very close to each other and the earthing resistance at station 3 is larger than at station 5, so a large part of the current injected at station 3 has an easy path to go into (from) the Earth at station 5. The only non-zero elements in columns 16 and 17 (not shown in Table 5) of the matrix $\mathbf{C}$ are the ones on the diagonal. This results from the zero earthing resistances at stations 16 and 17, which lead to the direct flow of the "pe" currents injected at these stations to (from) the Earth at the same sites. It is, however, worth noting that as the rows 16 and 17 also have non-zero off-diagonal elements, the currents $I_{\mathrm{e}, 16}$ and $I_{\mathrm{e}, 17}$ receive contributions from other injections as well. (Note the comment of the nature of $I_{\mathrm{e}, 16}$ and $I_{\mathrm{e}, 17}$ in Section 4.)

In general, the value of an element away from the diagonal becomes small in the matrix $\mathbf{C}$. This result, together with the numbering of the stations shown in Fig. 2, which implies that a small difference in the numbers generally means that the particular stations do not lie in very distant areas of the grid, indicates that remote parts of the network do not interact to any great extent. In other words, if earthing GIC values at a particular station are of interest, then it is sufficient to just take into account the neighborhood of the station. As an example, we consider the tenth row of the matrix $\mathbf{C}$, which gives the value of $I_{\mathrm{e}, 10}$ in terms of the "pe" currents $J_{\mathrm{e}, i}(i=1, \ldots, 17)$. (We could, of course, consider any other row as well, but this particular row is chosen just because it refers to the station Huutokoski, at which Finnish GIC recordings were started in the 1970s (Pirjola, 1983).) Table 6 shows $C(10, j)(j=1, \ldots, 17)$ arranged in order with its value. The corresponding value of $j$ and the distance between stations 10 and $j$ as well as a "neighborhood number" are also given. The neighborhood number is the number of stations from station 10 to station $j$ : it is 1 for the nearest stations, 2 for the second nearest stations, and so on. We clearly see that the elements $C(10, j)$ generally decrease with both an increasing distance and an increasing neighborhood number. A closer look at Table 6 reveals that the neighborhood number plays a more important role for the elements $C(10, j)$ than the distance. Consequently, only the nearby part of the grid affects GICs at station 10. This result is in full agreement with the conclusion presented by Pirjola (2005a), who considered an idealized linear network and the Finnish $400-\mathrm{kV}$ power grid.

The matrix $\mathbf{C}=\left(\mathbf{1}+\mathbf{Y}_{\mathrm{n}} \mathbf{Z}_{\mathrm{e}}\right)^{-1}$ has no explicit dependence on the distances between the stations or on the neighborhood numbers, but the influences come by the resistances $R_{i}^{\mathrm{e}}(i=1, \ldots, 17)$ and $R_{i m}^{\mathrm{n}}(i, m=1, \ldots, 17)$. If the neighborhood number between two stations becomes large, the "pe" current injected at one of them mostly goes into (from) the Earth before it reaches the other. This makes the corresponding element of the matrix $\mathbf{C}$ small. In any case, the currents always find the paths with the smallest resistance, which may lead to an explanation, for example, for the smaller value of $C(10, j)$ for $j=8$ than for $j=3$ or 15 even though the neighborhood number of station 8 is less than that of stations 3 and 15. A large part of the injected current $J_{\mathrm{e}, 8}$ goes, in addition to station 8 itself, to (from) the Earth at station 7 with a small earthing resistance (Table 1). This explanation is supported by the large element $C(7,8)$ (Table 5). I have conducted this study using station 10 , but the conclusions are clearly more generally valid. The earthing impedance matrix $\mathbf{Z}_{\mathrm{e}}$ is assumed to be diagonal in these calculations, but it is an irrelevant issue in this study because Pirjola (2008a, b) show that the off-diagonal elements of $\mathbf{Z}_{\mathrm{e}}$ are of minor importance in practice. Thus, the assumption of $\mathbf{Z}_{\mathrm{e}}$ to be diagonal obviously has no essential effect on the results in this paper.

\section{Concluding Remarks}

"Geomagnetically induced currents" in electric power transmission grids and in other conductor networks are produced by space storms that result from solar activity. Research on GICs has a lot of practical relevance because the GICs may cause problems to the systems and their operation. The GICs play a particularly important role at high latitudes, but GIC magnitudes and possible harmful effects also depend on the technological structures and details of the networks, so the GICs should be taken into account at lower latitudes as well. Furthermore, the continuously increasing dependence of people on reliable technology emphasizes the practical significance of the GICs and other space weather issues.

Modeling of the GICs in a network is usually carried out in two parts: (1) the determination of the horizontal (geo)electric field at the Earth's surface and (2) the computation of GICs driven by the field. The first part, which requires space physical and geophysical data and models, is more demanding and can be performed in practical terms only approximately. The second part is based on electric circuit theory and is exact in principle, but in the case of 
a complex network, in practice, assumptions are necessary for simplification. In this paper, I discuss the second part for an electric power transmission grid that is discretely earthed at transformer neutrals. Convenient matrix-type equations exist for the computation of both the earthing GICs to (from) the Earth at the stations and the GICs flowing in the transmission lines.

The standard formulation includes the (geo)voltages obtained by integrating the geoelectric field along the paths defined by the transmission lines. In this paper, I derive a matrix equation that directly couples the earthing GIC to the horizontal geoelectric field. On one hand, such a formulation can be considered to be simple and straightforward but, on the other hand, the transfer matrix is complicated because it is affected both by the numerical computation of the geovoltages and by the resistances of the system. The separation of these two contributions is obviously not easy in practice.

This paper also provides new theoretical and numerical analyses of the properties of the transfer matrix between the geovoltages and the earthing GICs in a power system. A particular result, which is important in practice, is that the ratio of the GICs flowing to (from) the Earth at a station under two different geophysical situations is independent of the total earthing resistance at this station. The studies made in this paper aim at a better understanding of the physical processes associated with the GICs. This is particularly achieved when describing the flow of GICs by assuming that the geoelectric field is replaced by the injection of "pe" currents into the network. An important result obtained by analyzing the behavior of the elements of the transfer matrix in this paper is that, when considering GICs at one site, model calculations can be limited to a smaller grid around the site. This is a confirmation of earlier conclusions drawn in a different way. Numerical computations discussed in this paper refer to an old configuration of the Finnish 400$\mathrm{kV}$ electric power transmission grid. I believe that this particular system is useful for testing different GIC calculation techniques, algorithms, and programs.

Acknowledgments. The author wishes to thank Dr. Ari Viljanen (Finnish Meteorological Institute) and Mr. Magnus Wik (Swedish Institute of Space Physics) for several inspiring and useful discussions associated with the items included in this paper and for the collaboration in the GIC research. The many valuable, careful, and constructive comments by the referees of the manuscript are gratefully acknowledged. A revision of the manuscript according to the referees' suggestions clearly improved and clarified the paper.

\section{References}

Bolduc, L., GIC observations and studies in the Hydro-Québec power system, J. Atmos. Sol.-Terr. Phys., 64(16), 1793-1802, 2002.

Boteler, D. H. and R. J. Pirjola, Modelling Geomagnetically Induced Currents produced by Realistic and Uniform Electric Fields, IEEE Trans. Power Delivery, 13(4), 1303-1308, 1998.

Boteler, D. H. and R. Pirjola, GIC Modelling, Technical Report for RealTime GIC Simulator, ESA Space Weather Pilot Project, ESTEC Contract Number 16986/03/NL/LvH, European Space Agency (ESA), 27 pp., 2004.
Boteler, D. H., R. J. Pirjola, and H. Nevanlinna, The effects of geomagnetic disturbances on electrical systems at the Earth's surface, Adv. Space Res., 22(1), 17-27, 1998.

Elovaara, J., P. Lindblad, A. Viljanen, T. Mäkinen, R. Pirjola, S. Larsson, and B. Kielén, Geomagnetically induced currents in the Nordic power system and their effects on equipment, control, protection and operation, CIGRÉ General Session 1992, (CIGRÉ= Inter-national Conference on Large High Voltage Electric Systems), Paris, France, August 31-September 5, 1992, Paper No. 36-301, 10 pp., 1992.

Kappenman, J. G., Geomagnetic storms and their impact on power systems, IEEE Power Eng. Rev., May 1996, 5-8, 1996.

Kappenman, J. G., An Overview of the Increasing Vulnerability Trends of Modern Electric Power Grid Infrastructures and the potential consequences of Extreme Space Weather Environments, in Effects of Space Weather on Technology Infrastructure, edited by I. A. Daglis, 257-286, NATO Science Series, Kluwer Academic Publishers, II. Mathematics, Physics and Chemistry, 176, Chapter 14: Space Weather and the Vulnerability of Electric Power Grids, 2004.

Kappenman, J. G. and V. D. Albertson, Bracing for the geomagnetic storms, IEEE Spectrum, March 1990, 27-33, 1990.

Lanzerotti, L. J., D. J. Thomson, and C. G. Maclennan, Engineering issues in space weather, in Modern Radio Science 1999, edited by M. A. Stuchly, 25-50, International Union of Radio Science (URSI), Oxford University Press, 1999.

Lehtinen, M. and R. Pirjola, Currents produced in earthed conductor networks by geomagnetically-induced electric fields, Ann. Geophys., 3(4), 479-484, 1985.

Mäkinen, T., Geomagnetically induced currents in the Finnish power transmission system, Finnish Meteorological Institute, Geophysical Publications, No. 32, Helsinki, Finland, 101 pp., 1993.

Molinski, T. S., Why utilities respect geomagnetically induced currents, $J$. Atmos. Sol.-Terr. Phys., 64(16), 1765-1778, 2002.

Pirjola, R., Induction in power transmission lines during geomagnetic disturbances, Space Sci. Rev., 35(2), 185-193, 1983.

Pirjola, R., Effects of space weather on high-latitude ground systems, $A d v$. Space Res., 36(12), doi:10.1016/j.asr.2003.04.074, 2231-2240, 2005a.

Pirjola, R., Averages of geomagnetically induced currents (GIC) in the Finnish $400 \mathrm{kV}$ electric power transmission system and the effect of neutral point reactors on GIC, J. Atmos. Sol.-Terr. Phys., 67(7), 701708, 2005b.

Pirjola, R., Calculation of geomagnetically induced currents (GIC) in a high-voltage electric power transmission system and estimation of effects of overhead shield wires on GIC modelling, J. Atmos. Sol.-Terr. Phys., 69(12), 1305-1311, 2007.

Pirjola, R., Study of effects of changes of earthing resistances on geomagnetically induced currents in an electric power transmission system, $R a$ dio Sci., 43, RS1004, doi:10.1029/2007RS003704, 13 pp., 2008a.

Pirjola, R., Effects of interactions between stations on the calculation of geomagnetically induced currents in an electric power transmission system, Earth Planets Space, 60, 743-751, 2008 b.

Pirjola, R. and M. Lehtinen, Currents produced in the Finnish $400 \mathrm{kV}$ power transmission grid and in the Finnish natural gas pipeline by geomagnetically-induced electric fields, Ann. Geophys., 3(4), 485-491, 1985.

Pulkkinen, A., S. Lindahl, A. Viljanen, and R. Pirjola, Geomagnetic storm of 29-31 October 2003: Geomagnetically induced currents and their relation to problems in the Swedish high-voltage power transmission system, Space Weather, 3, S08C03, doi: 10.1029/2004SW000123, 19 pp., 2005.

Watermann, J., The magnetic environment-GIC and other ground effects, in Space Weather, Research Towards Applications in Europe, edited by J. Lilensten, Springer, Chapter 5.0, 269-275, 2007.

Wik, M., A. Viljanen, R. Pirjola, A. Pulkkinen, P. Wintoft, and H. Lundstedt, Calculation of geomagnetically induced currents in the $400 \mathrm{kV}$ power grid in southern Sweden, Space Weather, 6, S07005, doi:10.1029/2007SW000343, 11 pp., 2008.

R. Pirjola (e-mail: risto.pirjola@fmi.fi) 